\title{
3D Model of Generalized Power Law Blood Flow in a Stenosed Bifurcated Artery
}

\author{
Azim Azahari, Zuhaila Ismail* and Normazni Abdullah \\ Department of Mathematical Sciences \\ Faculty of Science, Universiti Teknologi Malaysia \\ 81310 UTM Johor Bahru, Johor, Malaysia \\ ${ }^{*}$ Corresponding author: zuhaila@utm.my
}

Article history

Received: 10 September 2017

Received in revised form: 19 November 2017

Accepted: 14 January 2018

Published on line: 1 June 2018

\begin{abstract}
Numerical simulation of the behaviour of blood flow through a stenosed bifurcated artery with the presence of single mild stenosis at parent artery is investigated. The flow analysis applies the incompressible, steady, three-dimensional Navier-Stokes equations for non-Newtonian generalized power law fluids. Behaviour of blood flow is simulated numerically using COMSOL Multiphysicsthat based on finite element method.The results showthe effect of severity of stenosis on flow characteristics such as axial velocity and its exhibit flow recirculation zone for analysis on streamlines pattern.
\end{abstract}

Keywords Non-Newtonian; stenosis; bifurcated artery; COMSOL multiphysics.

\section{Mathematics Subject Classification 47.15}

\section{Introduction}

The study on behaviour of blood flow in blood vessels is very important since it could provide better understanding of their connection with blood vessel relatedproblem. One of the most well-known illness associated with blood vessels is cardiovascular disease. Cardiovascular disease as defined by World Health Organization (WHO) is a problem that happened due to the failure of blood vessels in heart to function well. Particularly, this disease related to a process called atherosclerosis. Atherosclerosis is a condition where the artery having an abnormal obstruction in its lumen which cause the disruption of blood flow [1]. This obstruction is called a stenosis or a plaque. Stenosis is made up of the deposition of cholesterol or fatty material on the arterial wall [2]. The existence of stenosis would disturb the normal flow of blood in the artery and reduce the amount of blood transport to the targeted area. Eventually, the targeted area did not receive the required amount of oxygen and nutrient needed and it would cause disease such as heart attack, stroke and aneurysms.

Many research works involving experiments and model simulations brought up that these diseases involving blood vessels strongly influenced by hemodynamic pattern of the flow. Hemodynamic patterns such as low wall shear stress (WSS), secondary flows and recirculation plays 
important roles in the formation and progression of atherosclerotic lesion. It is deduced that such complex flow patterns commonly occur in arteries particularly near branching, bifurcation, sites of curvature or expansion of cross-section [3-4]. For instance, Seo [5] observed that the average WSS along the outer wall is 2.2 less than that along the inner wall. This proofs that geometry of the vessels is one of the contributing factor affecting the blood flow circulation.

Blood rheological model is another important aspect to be considered in blood flow model. There are two types of blood behaviour which is Newtonian and non-Newtonian since viscosity of blood varies with the shear rate. Generally, blood behaves as non-Newtonian fluid. However, in large arteries the shear rate of blood is higher than $100 \mathrm{~s}^{-1}$ where the viscosity is practically constant. Due to this, it is acceptable to assume the blood to behave as Newtonian fluid. A study by $\mathrm{Xu}$. and Collins [6] investigate the numerical simulation study of 3D model blood flow in arterial bifurcation using code ACT EC based on finite volume method. It demonstrates that the flow occurs in Newtonian and non-Newtonian can be distinguished mainly by recognizing their flow separation zone. The effect of different non-Newtonian rheological model was investigated by Neofytou and Tsangaris [7] involving the Casson, power law and Quemada models in stenosis and aortic aneurysm. Based on this study, power law shows different flow behaviour for both cases while Quemada and Casson model exhibit similar flow behaviour for stenosis case but different flow behaviour in aneurysm case.

To analyse the bifurcated artery blood flow model behaviour, researcher normally developed a two-dimensional (2D) or three-dimensional (3D) model. Chakravarty and Mandal [8] proposed a 2D geometry of bifurcated artery while employing the experimental data as a parameter in their analysis. A 3D model of bifurcated artery was studied by Gupta [9] assuming a complete modelin a reduced one-dimensional Navier-Stokes equation. The purpose of the study was to investigate the behaviour of blood flow with the existence of magnetic field. Generally, most of the study utilized either MRI or glass carotid bifurcation model in order toanalyse 3D model of blood flow in bifurcated artery [10-11].

Therefore, present study investigate the blood flow of bifurcated artery with the presence of mild stenosis by develop the 3D model using the exact parameter as proposed by Chakravarty and Mandal [8] in their 2D model. This is because 3D model is much more realistic to the actual shape of the artery. Besides, this could provide us with a more reliable result to the investigation on the behaviour of blood flow in constricted artery. The model was built using COMSOL Multiphysics 5.2 which is a finite element method based computation software. Besides, the model of blood flow is described to be steady, incompressible and assume to follow non-Newtonian Generalized power law characteristics of fluid rheology.

\section{Problem Formulation}

Assumptions are imposed to formulate the present stenosis analytically before the simulation. The assumption is given as follow

- The arteries that forming bifurcation are rigid circular cylinders of finite length and symmetrical about the trunk axis.

- The parent aorta possesses a single stenosis in its lumen. 
- Curvatures are introduced at the lateral junctions and the flow divider so that one can rule out the possibility of the presence of any discontinuity causing non-existent of separation zones.

\subsection{Model Construction}

Based on the assumption, the geometry of stenosis in the arterial segment is visualized in Figure 1.

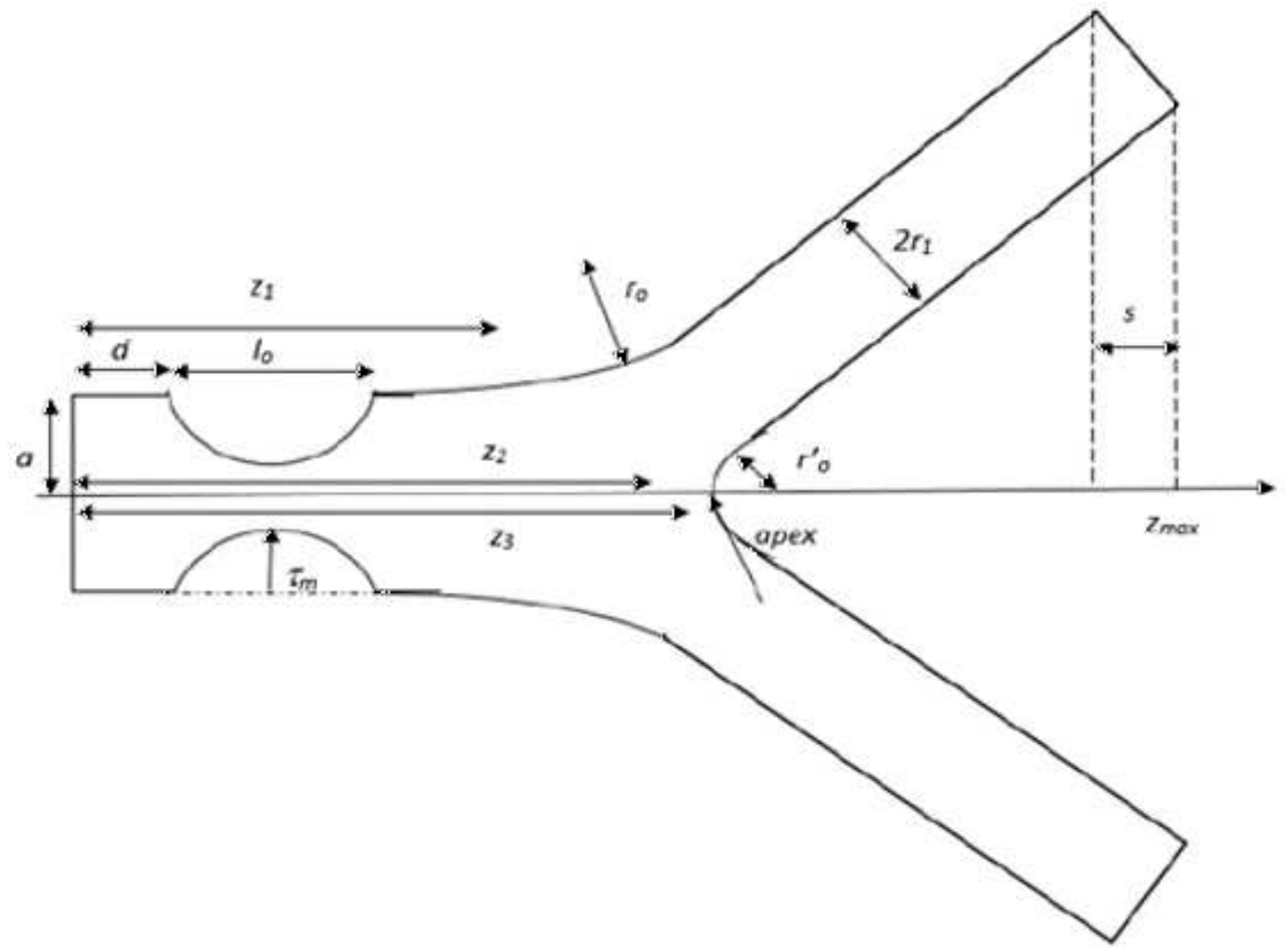

Figure 1: Schematic Model for Geometry of the Stenosed Arterial Bifurcation Model in the Plane at $z=0[8]$

In Figure 1, let $(x, y, z)$ be the coordinates of a material point in the Cartesian coordinate system where the $z$-axis is taken along the axis of trunk while $(x, y)$ are taken along the axial and radial directions respectively. The geometry of the bifurcated artery in the presence of stenosis is constructed mathematically of which the outer wall geometry is described by

$$
R_{1}(x)=\left\{\begin{array}{l}
a, 0 \leq x \leq d \text { and } d+l_{0} \leq x \leq x_{1}, \\
a+\frac{4 \tau m}{l_{0}^{2}}\left\{l_{0}(x-d)-(x-d)^{2}\right\}, d \leq x \leq d+l_{0} \\
a+r_{0}-\sqrt{r_{0}^{2}-\left(x-x_{1}\right)^{2}}, \mathrm{x}_{1} \leq x \leq x_{2}, \\
2 r_{1} \sec \beta+\left(x-x_{2}\right) \tan \beta, \mathrm{x}_{2} \leq x \leq x_{\max }-s
\end{array}\right.
$$


While at the daughter artery, the mathematical model is given by

$$
R_{2}(x)=\left\{\begin{array}{l}
0,0 \leq x \leq x_{3} \\
\sqrt{r_{0}^{\prime 2}-\left(x-\left(x_{3}+r_{0}^{\prime}\right)\right)^{2}}, \mathrm{x}_{3} \leq x \leq x_{4} \\
r_{0}^{\prime} \cos \beta+\left(x-x_{4}\right) \tan \beta, \mathrm{x}_{4} \leq x \leq x_{\max }
\end{array}\right.
$$

where $a$ and $r_{1}$ are the radii of the mother and daughter artery and $r_{0}, r_{0}^{\prime}$ are the radii of curvature for the lateral junction and the flow divider respectively. $l_{0}$ is the length of the stenosis at a distance $d$ from the origin, $x_{1}$ is the location of the onset of the lateral junction, $x_{2}$ is the offset of the lateral junction, $x_{3}$ is the apex, $\tau_{m}$ represents the maximum height of the stenosis at $d+\frac{l_{0}}{2}$ and $\beta$ is half of the bifurcation angle.

Moreover, the parameters involved in the above expressions (1) and (2) may be defined as

$$
\left.\begin{array}{c}
x_{2}=x_{1}+r_{0} \sin \beta, \\
r_{0}=\frac{a-2 r_{1} \sec \beta}{\cos \beta-1},
\end{array}\right\}
$$

where $q$ is chosen to be small number lying in the range $0.001 \leq q \leq 0.005$ for compatibility of geometry and

$$
x_{4}=x_{3}+r_{0}^{\prime}(1-\sin \beta)
$$

\subsection{Governing Equations}

The streaming fluid representing blood in the arterial bifurcation is generally considered to be laminar and following non-Newtonian power law characteristics of fluid rheology. The governing equations for the steady, incompressible, three-dimensionalflow in cartesian coordinate system are given by

$$
\begin{gathered}
\frac{\partial u}{\partial x}+\frac{\partial v}{\partial y}+\frac{\partial w}{\partial z}=0 \\
u \frac{\partial u}{\partial x}+v \frac{\partial u}{\partial y}+w \frac{\partial u}{\partial z}=-\frac{1}{\rho} \frac{\partial p}{\partial x}-\frac{1}{\rho}\left[\frac{\partial \tau_{x x}}{\partial x}+\frac{\partial \tau_{y x}}{\partial y}+\frac{\partial \tau_{z x}}{\partial z}\right] \\
u \frac{\partial v}{\partial x}+v \frac{\partial v}{\partial y}+w \frac{\partial v}{\partial z}=-\frac{1}{\rho} \frac{\partial p}{\partial y}-\frac{1}{\rho}\left[\frac{\partial \tau_{x y}}{\partial x}+\frac{\partial \tau_{y y}}{\partial y}+\frac{\partial \tau_{z y}}{\partial z}\right] \\
u \frac{\partial w}{\partial x}+v \frac{\partial w}{\partial y}+w \frac{\partial w}{\partial z}=-\frac{1}{\rho} \frac{\partial p}{\partial z}-\frac{1}{\rho}\left[\frac{\partial \tau_{x z}}{\partial x}+\frac{\partial \tau_{y z}}{\partial y}+\frac{\partial \tau_{z z}}{\partial z}\right]
\end{gathered}
$$

where $\vec{u}$ is the velocity vector, $\rho$ is the blood density, $p$ is the pressure and $\tau$ is the stress tensor. According to power law, the relation of the stress tensor and the strain rate, $\dot{\gamma}$ is defined as

$$
\tau=m|\dot{\gamma}|^{n-1} \dot{\gamma}=m\left|\sqrt{\frac{1}{2}(\dot{\gamma}: \dot{\gamma})}\right|^{n-1} \dot{\gamma}
$$


where $\dot{\gamma}$ is the strain rate tensor and $m$ is the fluid consistency coefficient and $n$ is the flow behaviourindex.

The velocity boundary condition on the surface of the artery are taken to be the usual no slip condition. Radial velocities of the streaming blood along the axis of the bifurcated artery till its apex may be assumed to be equal zero. At the inlet, parabolic velocity profile corresponding to fully developed flow is applied. This is stated by Mamun et al. [12] as

$$
u=U \cdot\left(1-\frac{y^{2}+z^{2}}{x^{2}+y^{2}}\right), v=0, w=0, \text { at } x=0-a \leq y, z \leq a
$$

where $U$ is the instantaneous velocity. A traction-free condition for both velocities (vanishing normal and tangential forces) is assumed at the outlet.

$$
\tau=\sigma \cdot \mathbf{n}=0
$$

where $\tau$ is the surface traction, $\sigma$ is the Cauchy stress tensor and $\mathbf{n}$ is the vector normal to the surface. For pressure point constraint, we assumed that

$$
p=0 \text { at } x=y=0, z=-a .
$$

\section{Computational Mesh and Numerical Method}

The governing equations subjected to the boundary and initial condition mentioned above were computed using COMSOL Multiphysics. The three-dimensional model was first constructed via COMSOL based on the two-dimensional blood flow model as displayed in Figure 1.

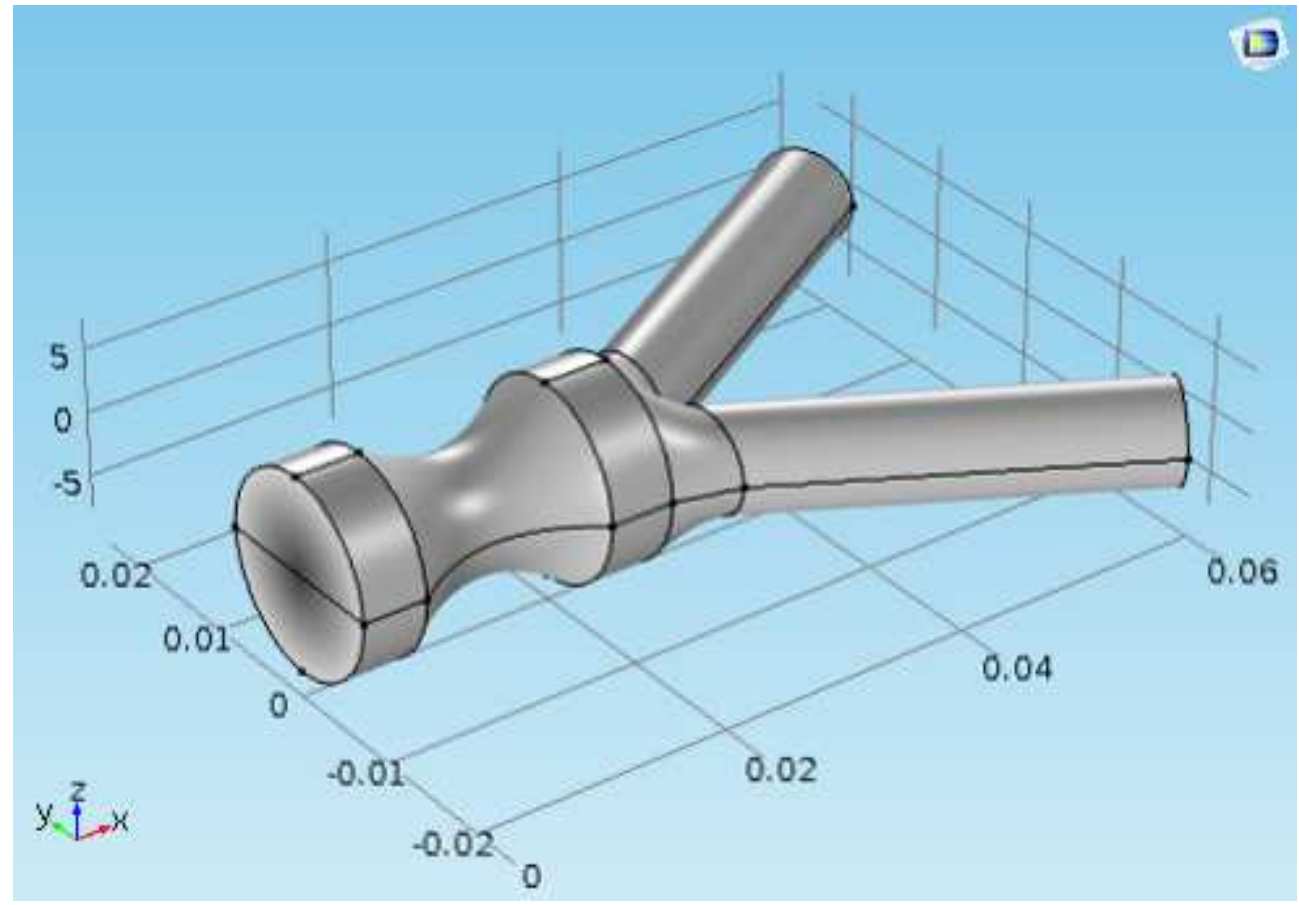

Figure 2: Three-dimensional Model of Bifurcated Artery with Mild Stenosis 
To validate the results, the 3D model in Figure 2 was converted into $2 \mathrm{D}$ model by cutting the model at $z=0$ into $x$ and $y$ plane. By using the same data proposed by Chakravarty and Mandal [8], the mesh test and maximum velocity at the region of mother artery can be determined and verified. The parameter values are: $a=0.0075 \mathrm{~m}, l_{0}=0.0015 \mathrm{~m}, d^{\prime}=0.005 \mathrm{~m}$, $z_{\text {max }}=0.006 \mathrm{~m}, \rho=1.05 \times 10^{3} \mathrm{kgm}^{-3}, \mu=0.035 \mathrm{P}, \beta=30^{\mathrm{O}}, z_{4}=0.0025 \mathrm{~m}, r_{1}=0.51 a, k=0.1$ and $z=0.0125 \mathrm{~m}$.

The mesh dependency test was conducted to ensure the results were not dependent on the mesh parameters. This was done by generating the unstructured triangular elements of the model by using built-in meshing function. The discretized geometry is shown in Figure 3. Several attempts had been made but only four of them will be stated here. The number of domain elements, domain boundaries and degree of freedom for each 2D mesh test are summarized in Table 1.

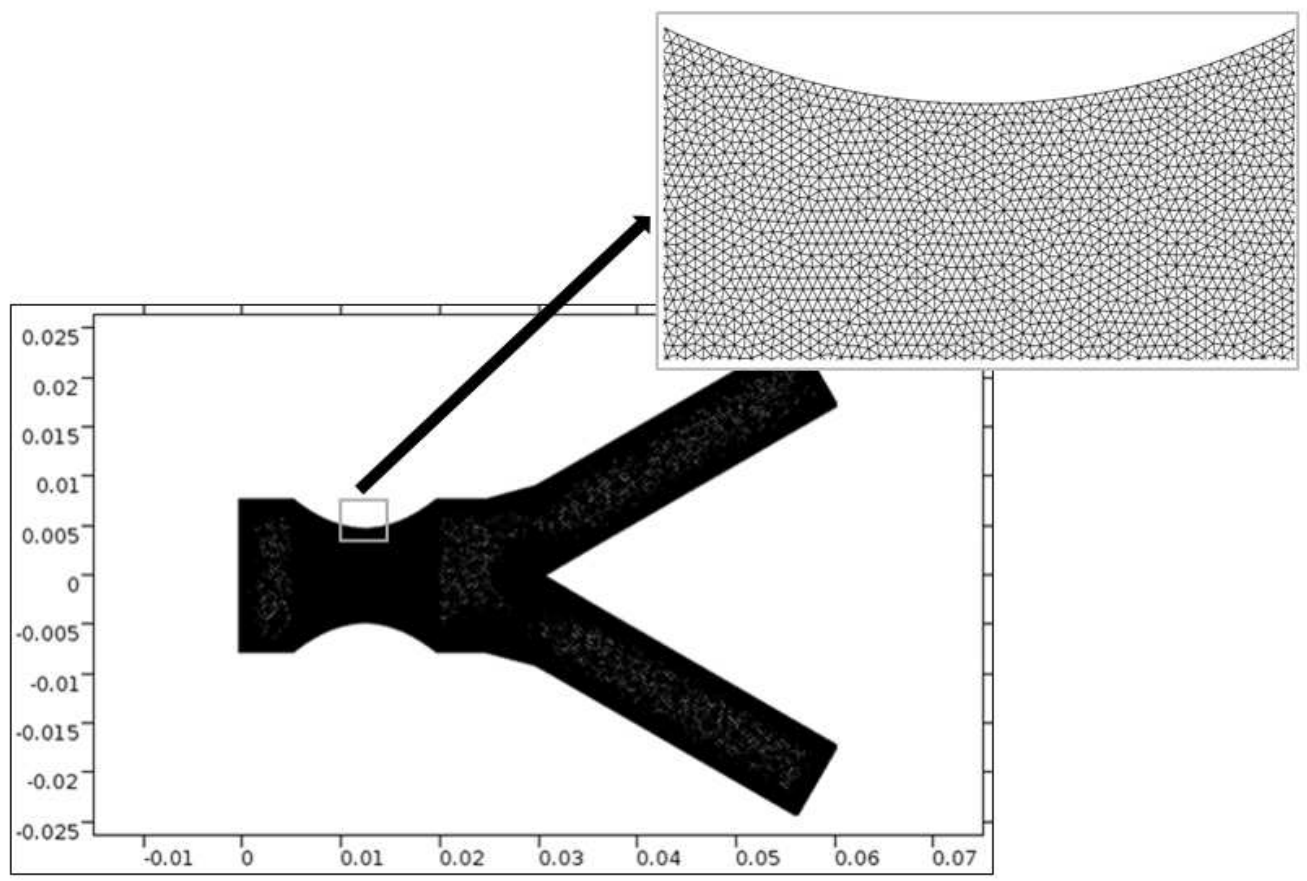

Figure 3: Unstructured Triangular Mesh Elements for 2D Bifurcated Artery for Mesh 3

Table 1: Mesh Parameters for 2D Geometry Bifurcated of Artery

\begin{tabular}{|c|c|c|c|}
\hline Parameter & Domain Elements & Boundary Elements & Degree of Freedom \\
\hline Mesh 1 & 27365 & 720 & 42117 \\
\hline Mesh 2 & 39844 & 718 & 60846 \\
\hline Mesh 3 & 48933 & 1237 & 75258 \\
\hline Mesh 4 & 53178 & 1116 & 81444 \\
\hline
\end{tabular}

From each of the mesh, the results of velocity magnitude are evaluated and graphically shown in Figure 4. The results indicated that there is only very small variation between the 
results, particularly contributed by mesh 1 and mesh 2 . The velocity magnitude from mesh 3 at the axial distance where the constriction is maximum $(z=0.0125 \mathrm{~m})$ approximately the same as those of mesh 4 . Therefore, we decided that the parameters quantities of mesh 3 are sufficient to provide good solution to the problem.

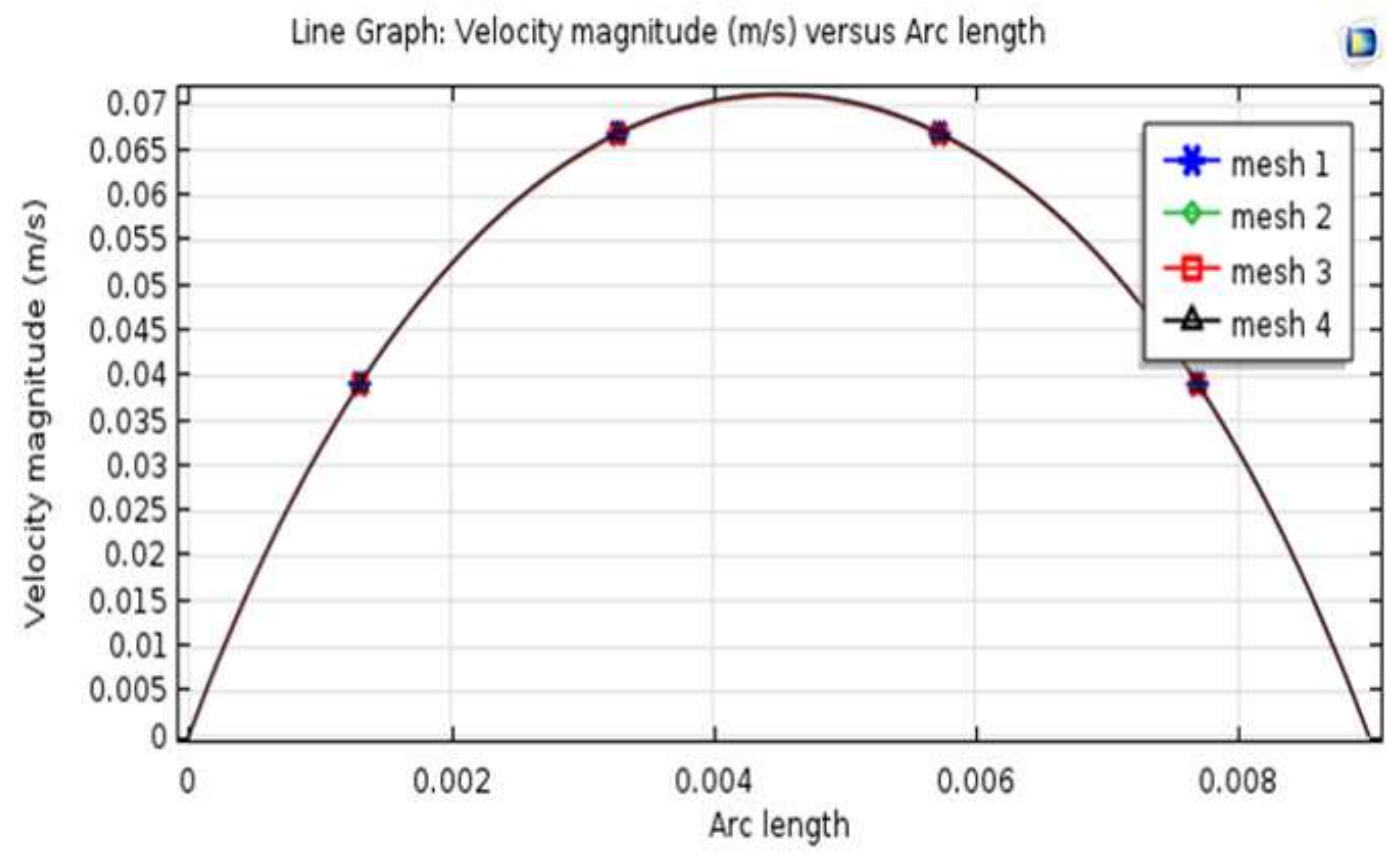

Figure 4: Velocity Magnitude for Different Mesh at $z=0.0125 \mathrm{~m}(2 \mathrm{D})$

Figure 5 displayed the results for velocity magnitude at $z=0.0125 \mathrm{~m}$ which is obtained by COMSOL Multiphysics 5.2 while figure 6 shows the velocity magnitude plotted by Ismail et al. [2] and Chakravarty and Mandal [8]. The outcome by COMSOL is achieved when the Reynolds number is set to 110.

Since Chakravarty and Mandal [8] using a dimensionless radial distance, hence the arc length from Figure 5 will be divided by 0.005 to obtain the same dimensionless radial distance. As the point to be observed is at 0.2 on Figure 6 hence the value 0.2 on Figure 5 is at arc length 0.001. It can be show as below:

$$
x=\frac{0.001 \mathrm{~m}}{0.005 \mathrm{~m}}=0.2
$$

Both result is approximately the same and nearly achieve $0.07 \mathrm{~ms}^{-1}$. The graph plot is then slowly decreasing to zero, as the flow reach the wall of the artery. The error between the results is very small with $0.003 \mathrm{~ms}^{-1}$ and $0.002 \mathrm{~ms}^{-1}$ which is due to different techniques and inlet velocity chosen to solve the problem. The study was then proceeded by running the mesh test for 3D blood flow model.

For three-dimensional blood flow model, the Reynolds number, Re and power law index, $n$, are based on the research by Chakravarty and Mandal [8] which are given as $\mathrm{Re}=300$ and $n=0.639$. Although several attempts had been made, only nine were selectedto be shown in this paper. The geometry of the discretized 3D bifurcated artery and the results of the mesh dependency which illustrated by velocity magnitude profile is shown in Figure 7 and 8 


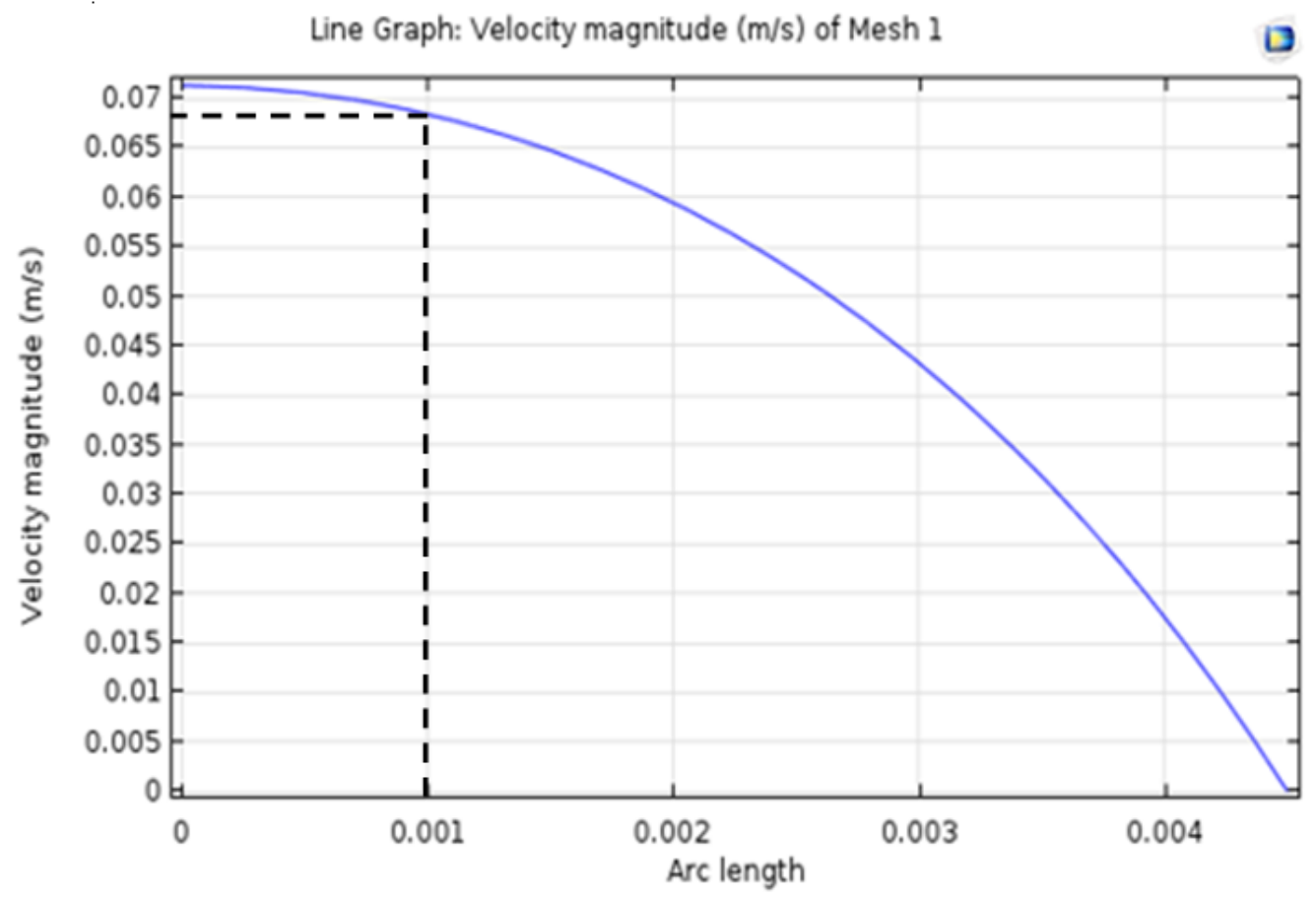

Figure 5: The Axial Velocity at $z=0.0125 \mathrm{~m}$ as Obtained from COMSOL Multiphysics

respectively. The velocity magnitude from mesh 5 at the axial distance where the constriction is maximum $z=0.0125 \mathrm{~m}$ approximately the same as those for mesh 8 . This suggests that parameters quantities of mesh 5 are sufficient to provide good solution for the problem.

\section{Results and Discussion}

The study of three-dimensional blood flow model was conducted based on the parameters proposed by Chakravarty and Mandal [8] as mention in previous section. The main purpose of this study was to investigate the effect of different stenosis severity to the blood flow and analysing the streamlines pattern of the flow. The location of the observation is $0.0125 \mathrm{~m}$ from the inlet boundary, $x=0.0125 \mathrm{~m}$ and $0.005 \mathrm{~m}$ from the junction of the daughter artery. Hence, the location can be illustrated as Figure 9.

The study considers maximum height used by Mustapha et al. [13], 40\% occlusion and three other maximum height of stenosis as proposed by Tan and Mustapha [14] where the severity of the stenosis was set by modifying the geometry to have $48 \%, 75 \%$ and $87 \%$ occlusion in the constricted arterial segment. The different maximum height of stenosis is shown in Figure 10. The resultant velocity profiles and streamlines were discussed below.

Figure 11 designates the different in stenosis severity effects on velocity magnitude at $x=$ $0.0125 \mathrm{~m}$. Notice that, at $40 \%$ occlusion the severity of stenosis is at its lowest and gradually increase as the severity percentage increase. This happens since the radius of artery will become smaller as the stenosis becomes more severe. As a result, the velocity of flow across the stenosis will increase. Hence, this could indicate that the presence of stenosis with different severity could disturb the normal velocity profile of blood. 


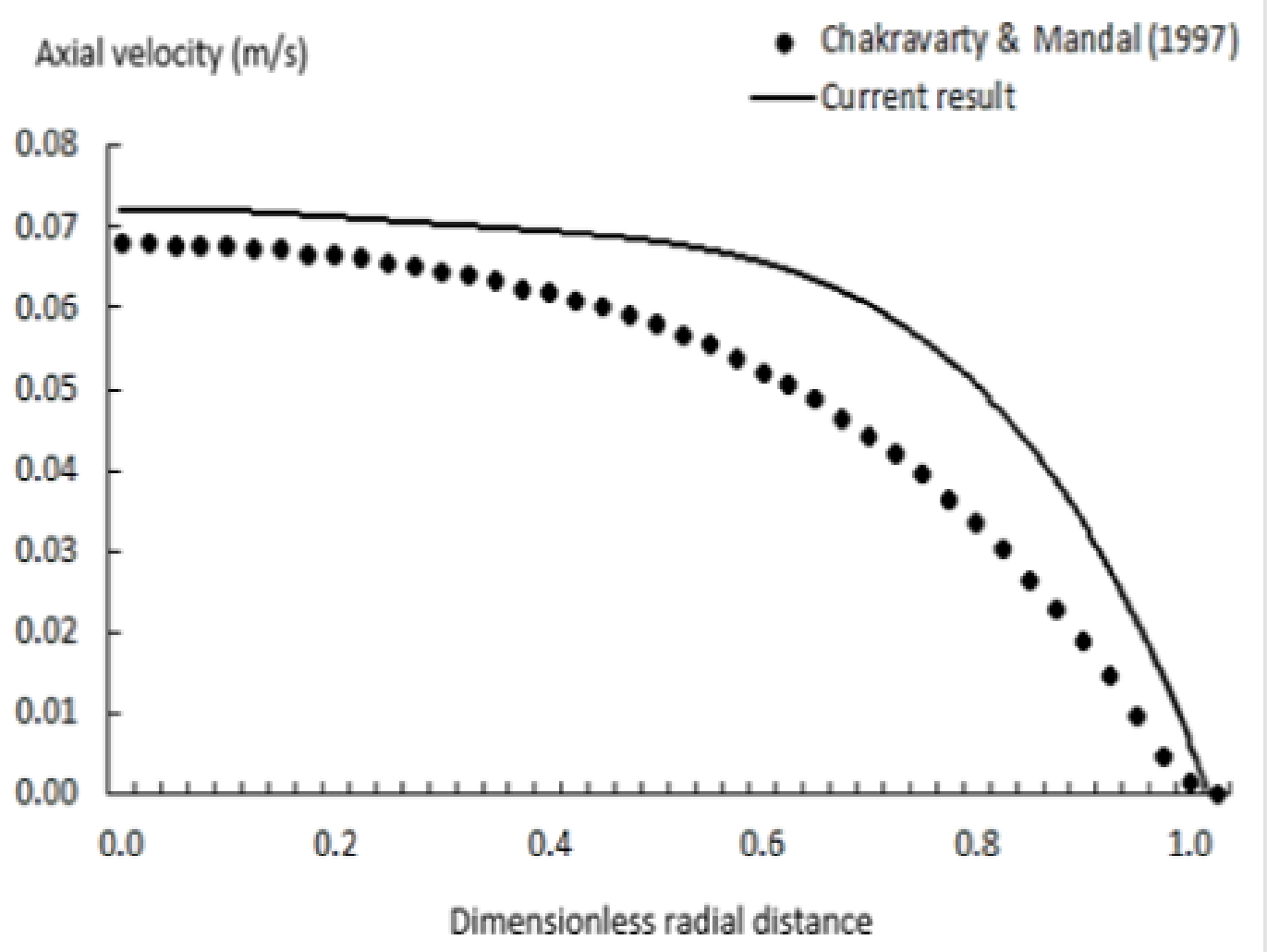

Figure 6: The Axial Velocity at $z=0.0125 \mathrm{~m}$ as Obtained by Ismail et al. [2] and Chakravarty and Mandal [8]

The role of stenosis severity on blood flow problem were further visualized by analysing the streamlines of the upper half of the bifurcated artery. However, the 3D model cross section will be used since the streamlines results cannot be seen clearly if taken directly from 3D model. Streamlines results for 40\%, 48\%, 75\% and 87\% occlusion are presented in Figure 12, Figure 13, Figure 14 and Figure 15 respectively.

Figure 12 where the severity of stenosis at $40 \%$, the streamlines shows the behaviour of an abnormal flow with the presence of the recirculation zone near the offset of the stenosis. As the severity increase to $48 \%$, the recirculation zone starts to elongate along the wall of daughter artery. The recirculation zone gets worse when the percentage of severity of stenosis at $75 \%$ where the flow starts to turn into turbulent flow when its starts to enter the daughter artery. Further increment of stenosis occlusion at $87 \%$ cause the turbulent flow to be fully developed in the daughter artery.

\section{Conclusion}

The main purpose of this study is to investigate the influence of the severity of stenosis on velocity profile and streamlines pattern of non-Newtonian blood flow in a 3D bifurcated artery. The velocity profiles and streamlines patterns for different severity of stenosis have been plotted and presented. From the results, it can be concluded that increase in severity of stenosis causes the axial velocity of blood to increase as well. Furthermore, more circulation will appear at 
Table 2: Mesh Parameters for 3D Geometry Bifurcated of Artery

\begin{tabular}{|c|c|c|c|}
\hline Parameter & Domain Elements & Boundary Elements & Degree of freedom \\
\hline Mesh 1 & 62675 & 5986 & 48408 \\
\hline Mesh 2 & 108626 & 9024 & 82276 \\
\hline Mesh 3 & 308721 & 22524 & 229728 \\
\hline Mesh 4 & 182319 & 9768 & 132388 \\
\hline Mesh 5 & 218310 & 12624 & 159504 \\
\hline Mesh 6 & 457627 & 25822 & 332828 \\
\hline Mesh 7 & 584616 & 22238 & 414496 \\
\hline Mesh 8 & 782837 & 34368 & 559252 \\
\hline Mesh 9 & 3076758 & 68854 & 2128856 \\
\hline
\end{tabular}

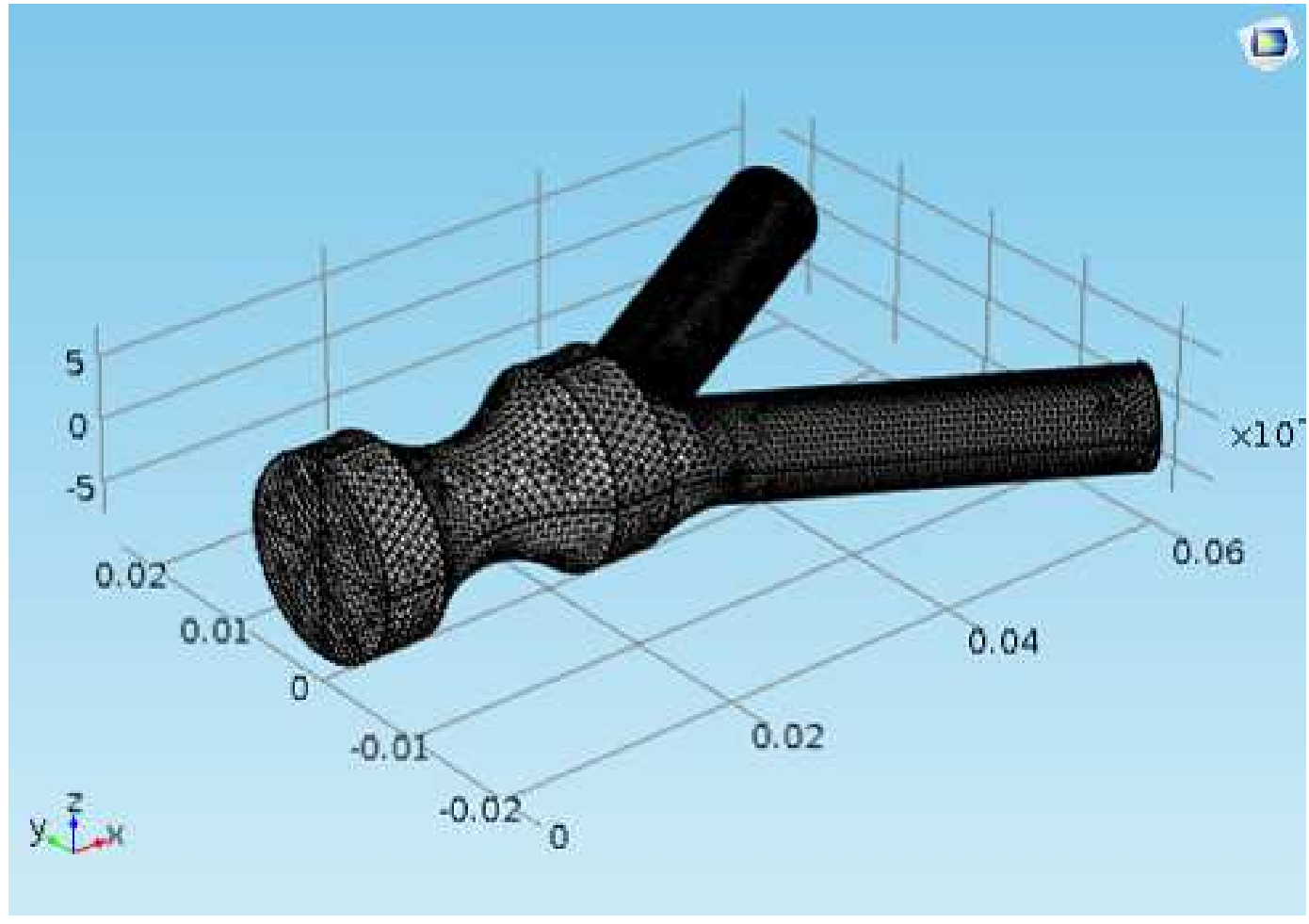

Figure 7: Unstructured Triangular Mesh Elements for 3D Bifurcated Artery 


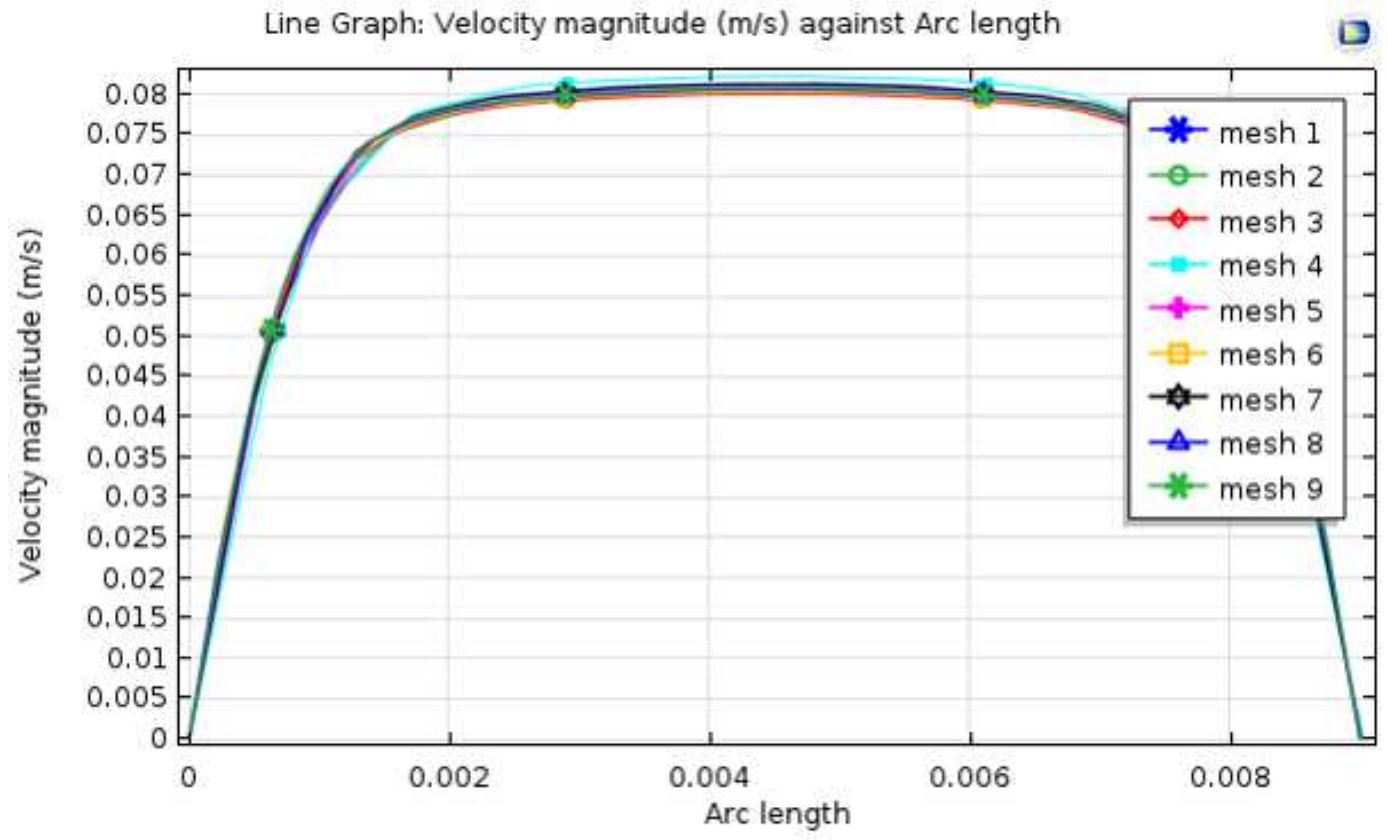

Figure 8: Velocity Magnitude for Different Mesh at $z=0.0125 \mathrm{~m}$ (3D)

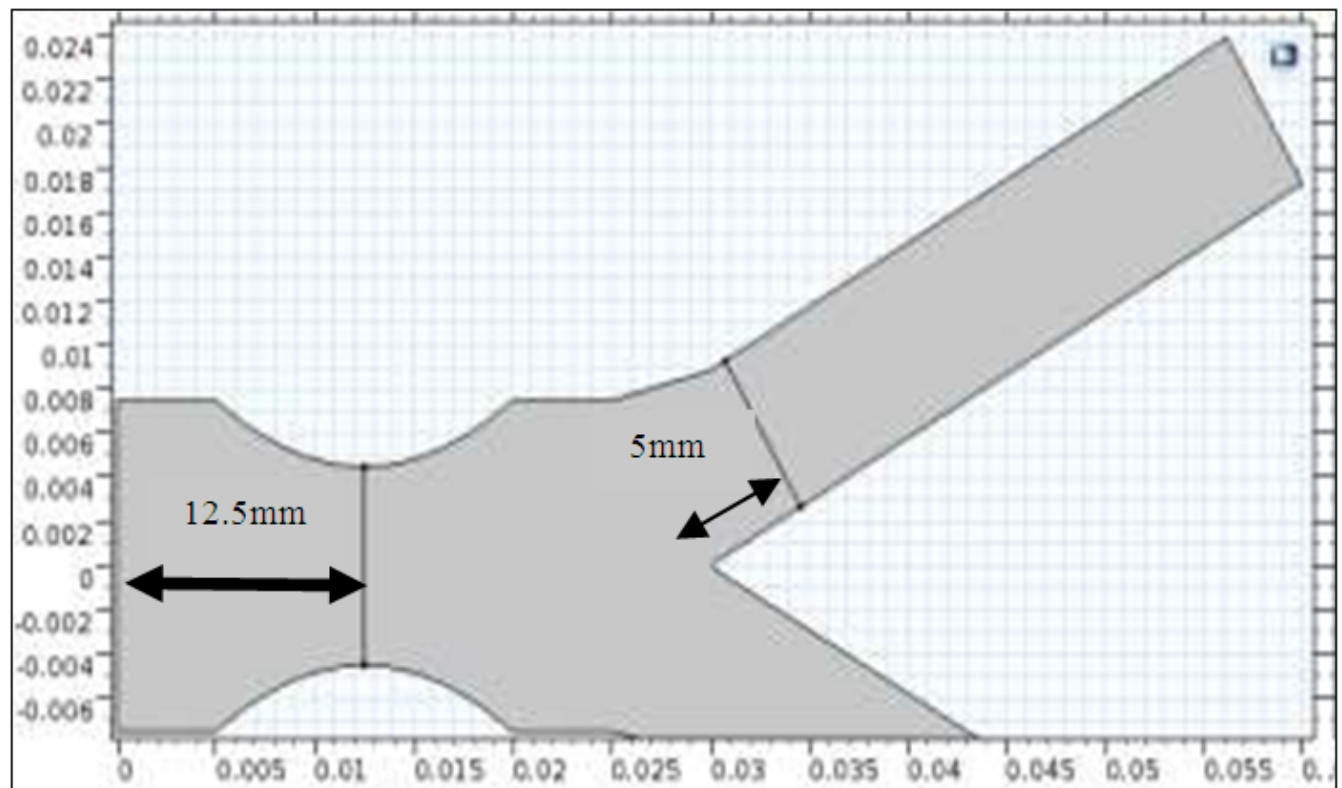

Figure 9: Location of the Evaluation of Problem Variables 


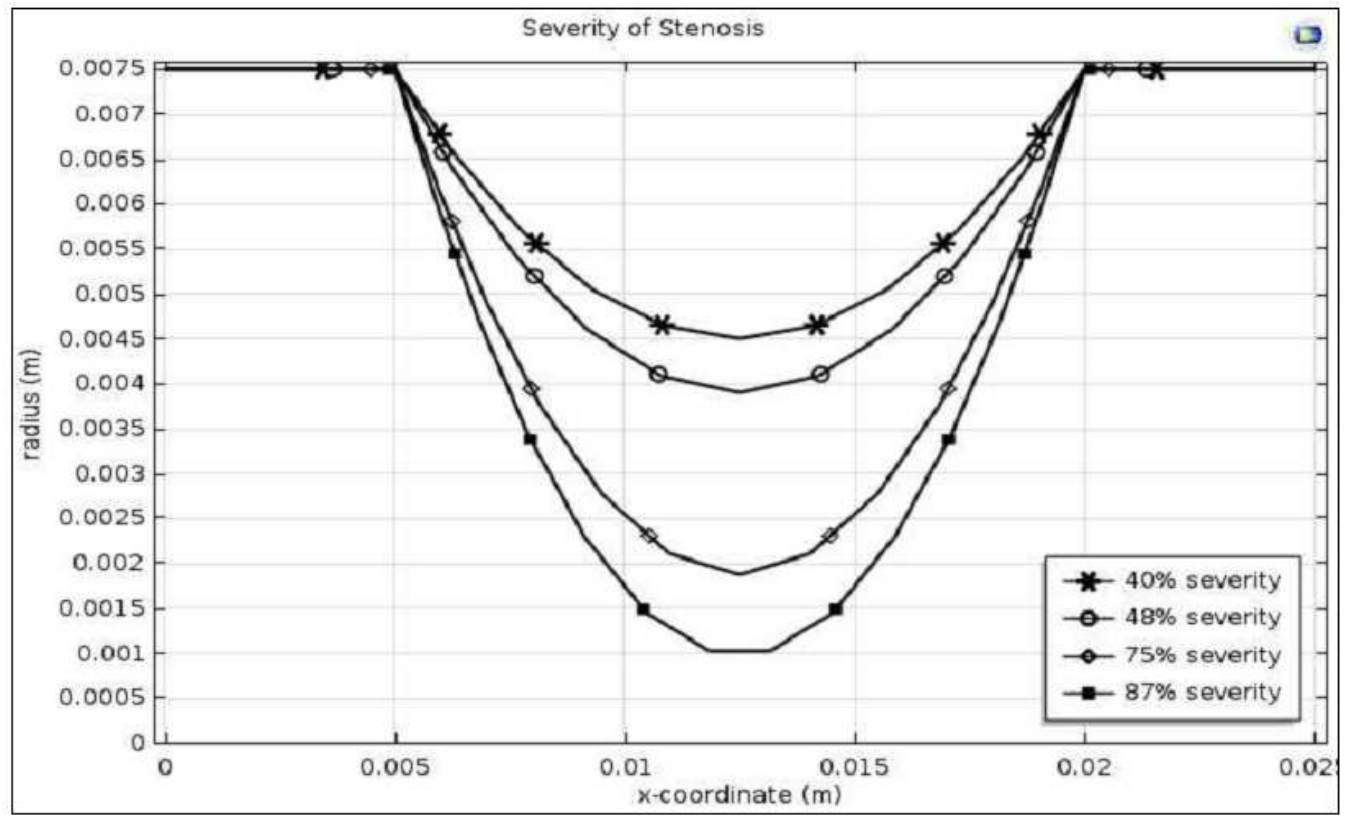

Figure 10: Different Severity of Stenosis

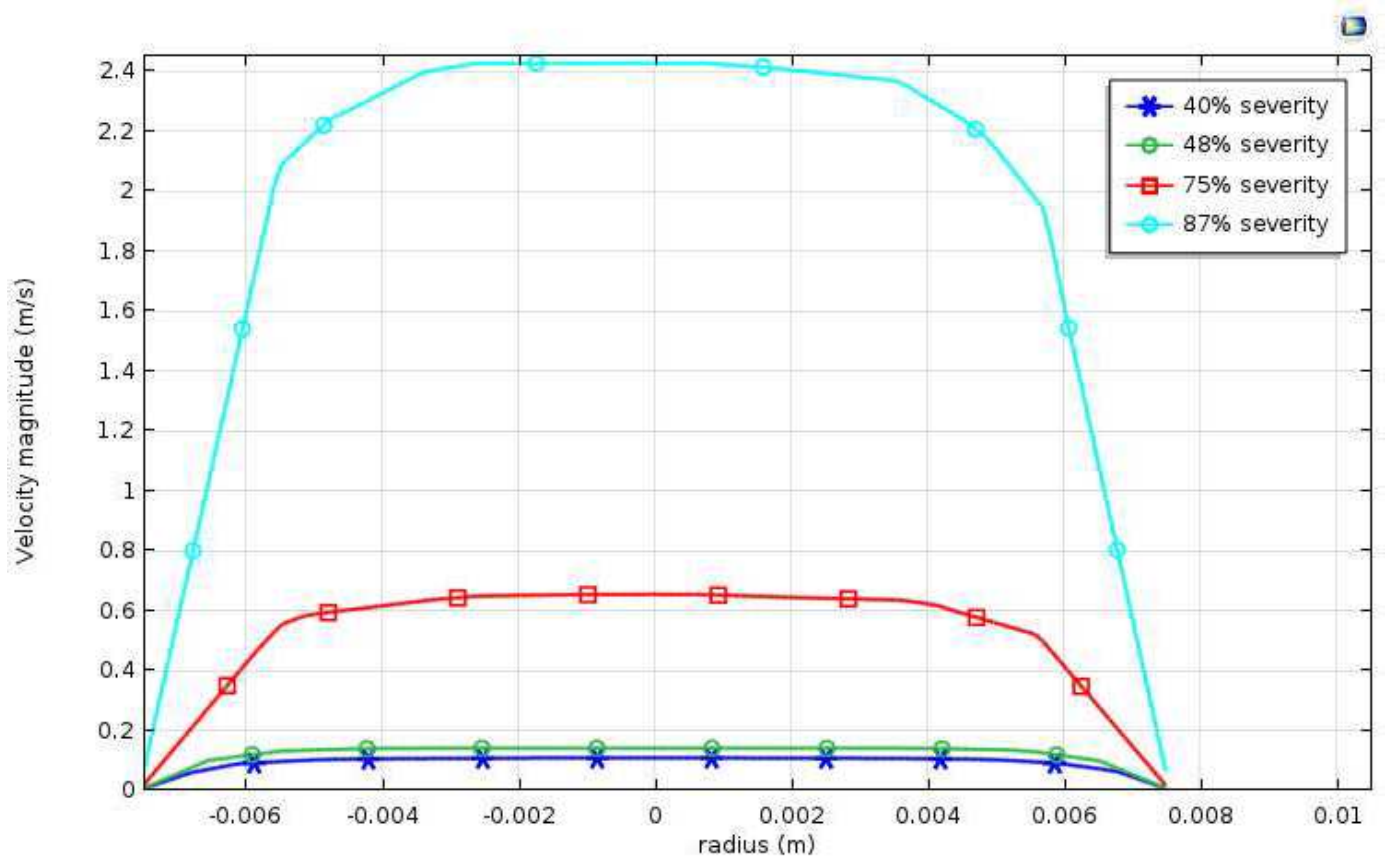

Figure 11: Velocity Magnitude at Different Severity of Stenosis 


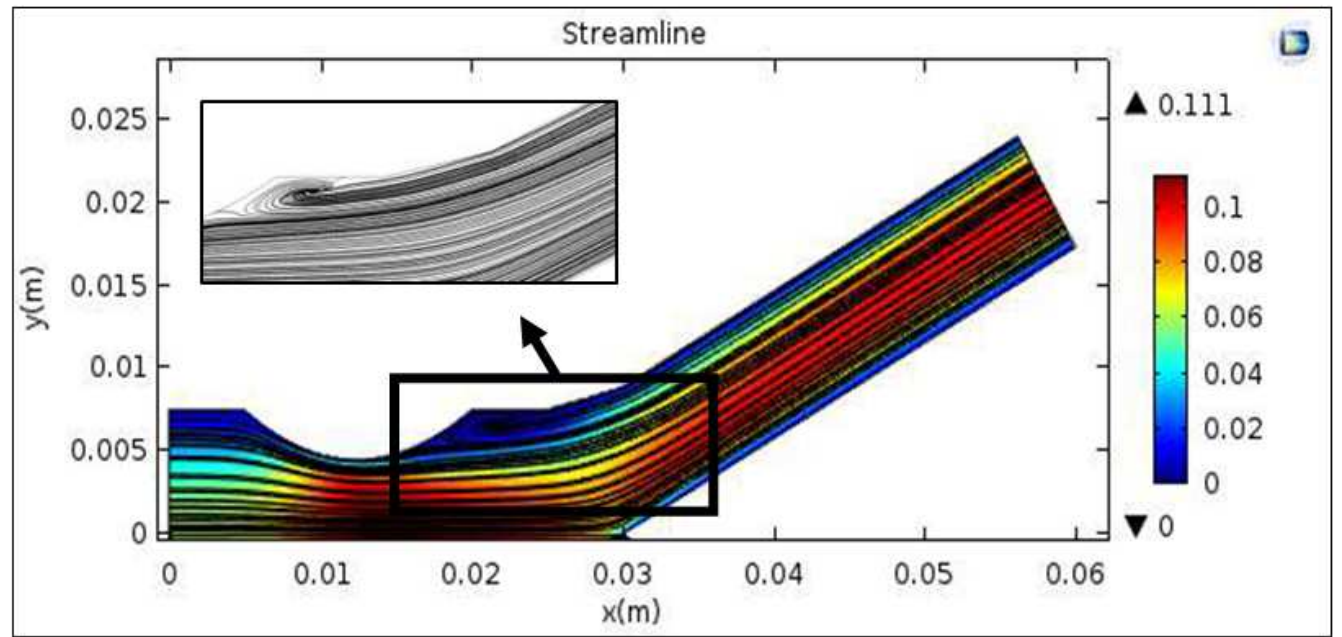

Figure 12: Streamlines Patterns of Blood Flow at 40\% stenosis

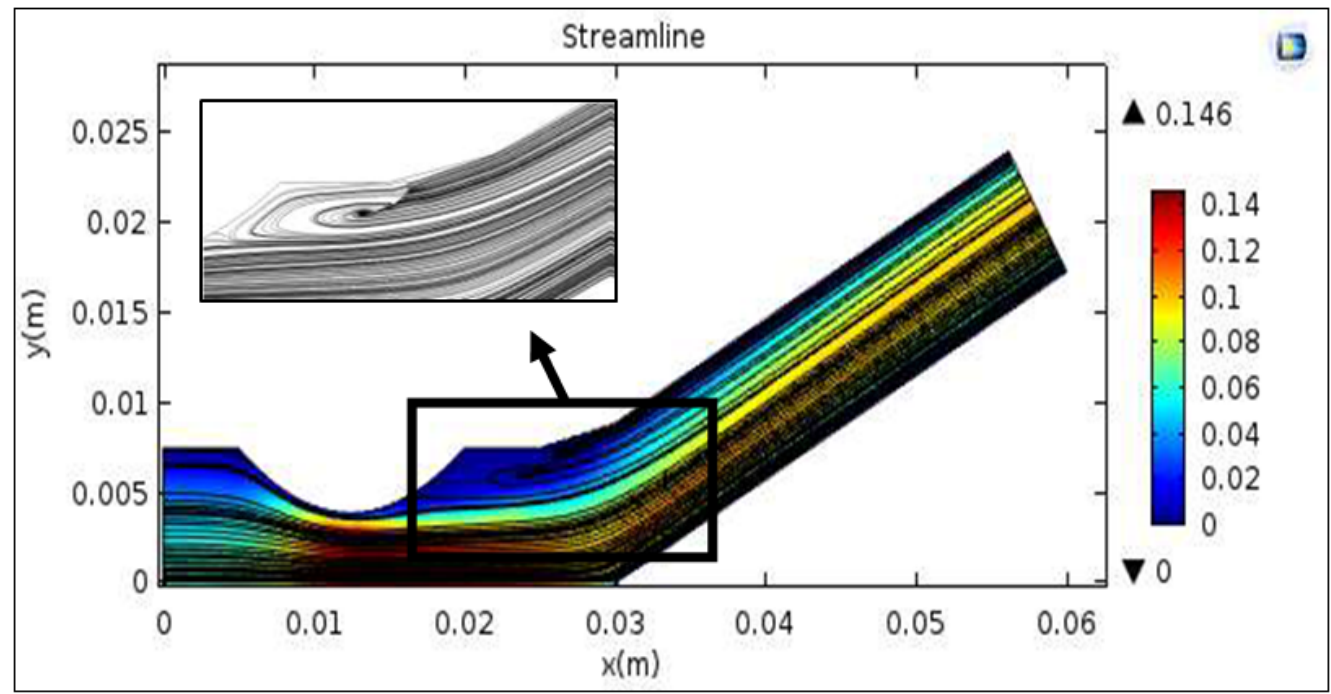

Figure 13: Streamlines Patterns of Blood Flow at $48 \%$ stenosis 


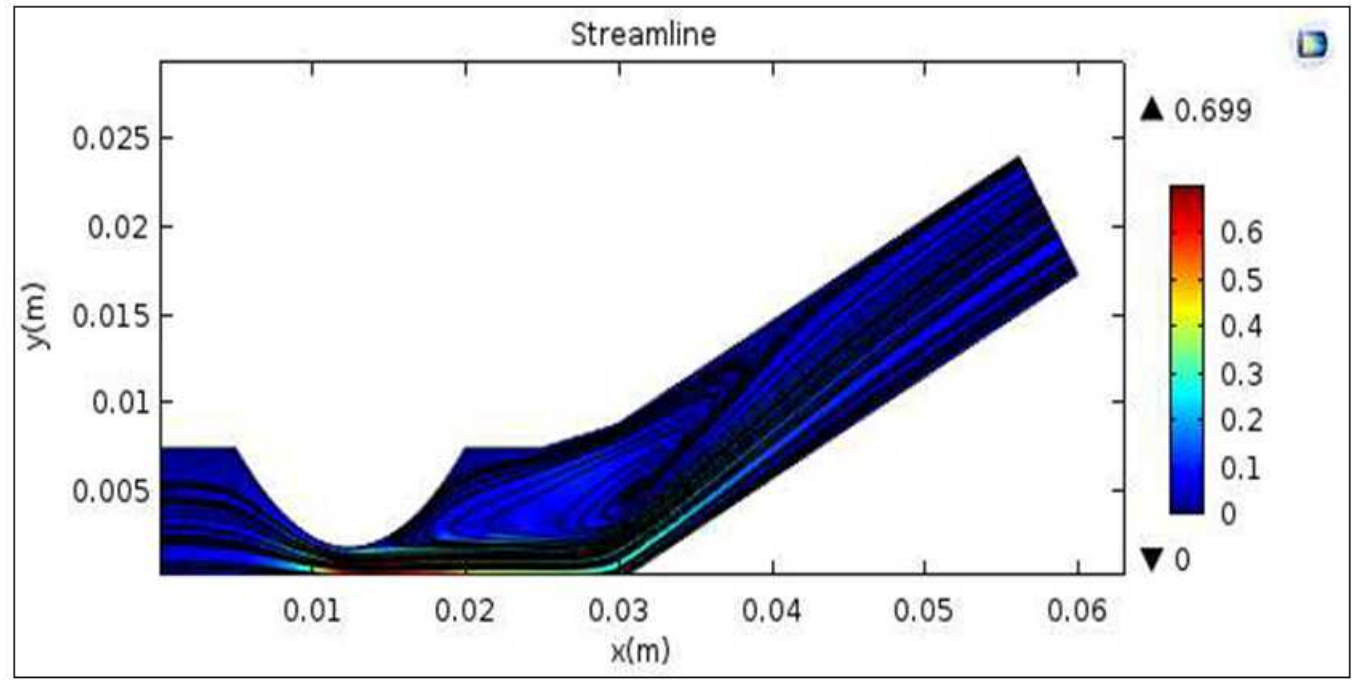

Figure 14: Streamlines Patterns of Blood Flow at 75\% Stenosis

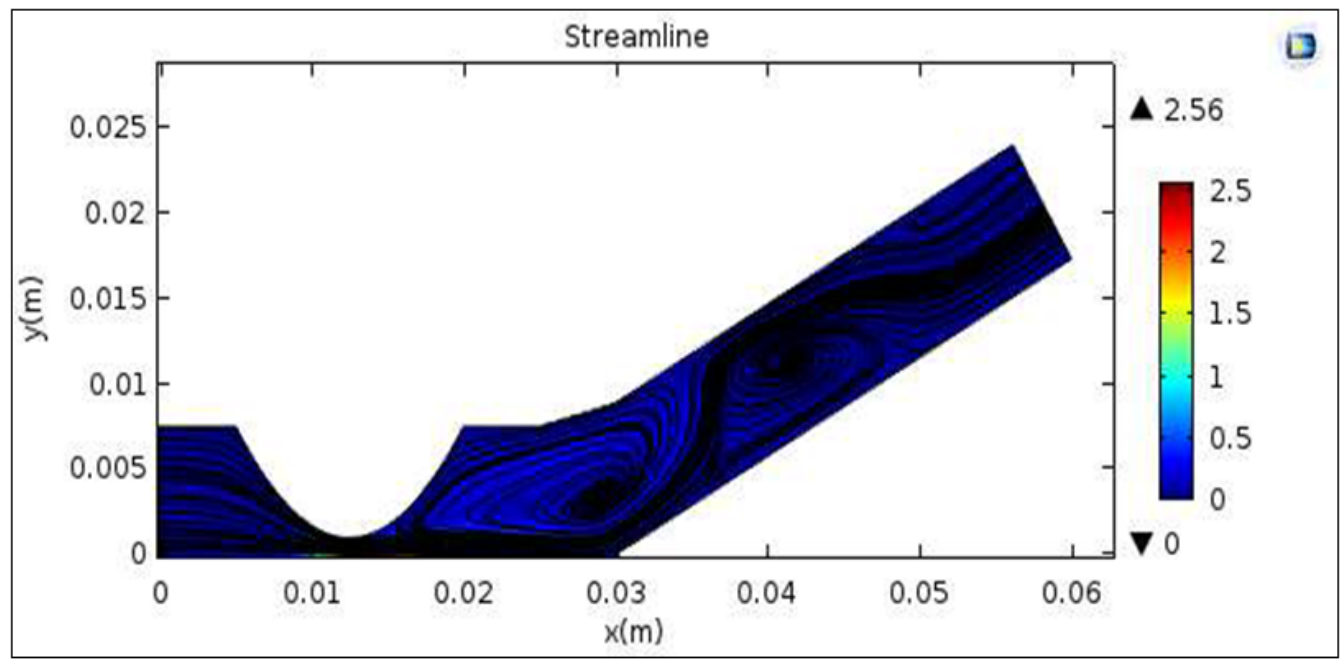

Figure 15: Streamlines Patterns of Blood Flow at 87\% Stenosis 
downstream of stenosis and along the edge of the daughter artery. From medical view, the existence of flow recirculation in cardiovascular system may cause danger to the health of patient particularly atherosclerosis patient since blood is moving very slowly during this state. Henceforth, more research in this area should be conducted to enhance our knowledge in arterial disease process and treatment.

\section{Acknowledgement}

The author would like to thank the Ministry of High Education (MOHE), STEM Grant with vote no. A. J091002.5600.07397 and Research Management Centre - UTM for the financial support through vote numbers 4F632,13H28 and $19 \mathrm{H} 00$ for this research.

\section{References}

[1] Huda Salmi Ahmad. A Generalized Power-law Model of Blood Flow Through Tapered Arteries with an Overlapping Stenosis. Universiti Teknologi Malaysia: Ph.D. Thesis. 2014.

[2] Zuhaila Ismail. Mathematical Modelling of Non-Newtonian Blood Flow through a Tapered stenotic artery. Universiti Teknologi Malaysia: Ph.D. Thesis. 2006.

[3] J. R. Lewick. An Introduction to Cardiovascular Physiology. London: Hodder Arnold Publishers. 2010.

[4] Zarins, C. K., Giddens, D. P., Bharadvaj, B. K., Sottiurai, V. S., Mabon, R. F. and Glagov, S. Carotid bifurcation atherosclerosis. Quantitative correlation of plaque localization with flow velocity profiles and wall shear stress. Circulation Research. 1983. 53: 502-514.

[5] Seo, T. Numerical simulations of blood flow in arterial bifurcation models. KoreanAustralia Rheology Journal. 2013. 25(3): 153-161.

[6] Xu, X. Y. and Collins, M. W. Studies of blood flow in arterial bifurcations using computational fluid dynamics. Journal of Engineering in Medicine.

[7] Neofytou, P. and Tsangaris, S. Flow effects of blood constitutive equations in 3D models of vascular anomalies. International Journal for Numerical Methods in Fluids. 51(5): 489-510.

[8] Chakravarty, S. and Mandal, P. K. An analysis of pulsatile flow in a model aortic bifurcation. International Journal of Engineering Science. 1997. 35(4): 409-422.

[9] Gupta, A. K. Performance and analysis of blood flow through carotid artery. International Journal of Engineering Business Management. 2011. 3(4): 1-6.

[10] Ku, D. N., Giddens, D. P., Zarins, C. K. and Glagov, S. Pulsatile flow and atherosclerosis in the human carotid bifurcation. Arteriosclerosis, Thrombosis, and Vascular Biology. 1985. 5(3): 293-302. 
[11] Milner, J. S., Moore, J. A., Rutt, B. K. and Steinman, D. A. Hemodynamics of human carotid artery bifurcations: Computational studies with models reconstructed from magnetic resonance imaging of normal subjects. Journal of Vascular Surgery. 1998. 28(1): $143-156$.

[12] Mamun, K., Akhter, M. N. and Ali, M. Physiological non-Newtonian blood flow through single stenosed artery. Theoretical and Applied Mechanics. 2016. 43(1): 99-115.

[13] Mustapha, N., Charavarty, S., Mandal, P.K. and Amin. N. Unsteady response of blood flow through a couple of irregular arterial constrictions to body acceleration. Journal of Mechanics in Medicine and Biology. 2008. 8(3):395-420.

[14] Tan, Y. B. and Mustapha, N. The gravitational effects of blood flow in irregular stenosed artery with various severity. Journal of Mathematical and Computational Science. 2016. 2(1): 28-39. 